\title{
ON THE CLUSTERING CONJECTURE FOR BERNOULLI FACTORS OF BERNOULLI SHIFTS
}

\author{
G. KELLER
}

(Communicated by J. J. Uhl, Jr.)

\begin{abstract}
We give sufficient algebraic conditions on the probabilities $p_{i}$ of a Bernoulli shift $B(\mathbf{p})=B\left(p_{1}, \ldots, p_{M}\right)$ which imply that if $B(\mathbf{q})=$ $B\left(q_{1}, \ldots, q_{N}\right)$ is a continuous factor of $B(\mathbf{p})$, then $\mathbf{q}$ is a clustering of $\mathbf{p}$.
\end{abstract}

Let $\mathbf{p}=\left(p_{1}, \ldots, p_{M}\right)$ and $\mathbf{q}=\left(q_{1}, \ldots, q_{N}\right)$ be probability vectors defining Bernoulli shifts $B(\mathbf{p})=\left(\{1, \ldots, M\}^{\mathbf{Z}}, \mathbf{p}^{\mathbf{Z}}, \sigma_{M}\right)$ and $B(\mathbf{q})=\left(\{1, \ldots, N\}^{\mathbf{Z}}, \mathbf{q}^{\mathbf{Z}}\right.$, $\left.\sigma_{N}\right)$. We assume throughout that $B(\mathbf{q})$ is a continuous factor of $B(\mathbf{p})$, i.e., there is a continuous homomorphism $\Phi$ from $B(\mathbf{p})$ to $B(\mathbf{q})$. (Homomorphism means that $\mathbf{p}^{\mathbf{Z}} \circ \Phi^{-1}=\mathbf{q}^{\mathbf{Z}}$ and $\Phi \circ \sigma_{M}=\sigma_{N} \circ \Phi$.) If $h(\mathbf{p})=-\sum_{i} p_{i} \log p_{i}$ denotes the entropy of $B(\mathbf{p})$, then $h(\mathbf{p}) \geq h(\mathbf{q})$.

Tuncel [4] and, independently, del Junco et al. [2] showed that if $h(\mathbf{p})=h(\mathbf{q})$, then $\mathbf{q}$ is just a permutation of $\mathbf{p}$, i.e., there is a trivial factor map from $B(\mathbf{p})$ onto $B(\mathbf{q})$. Note, however, that $\Phi$ need not be this trivial map! Del Junco et al. showed that if $\mathbf{p}=(1 / M, \ldots, 1 / M)$ and $h(\mathbf{p})>h(\mathbf{q})$, then $\mathbf{q}=$ $\left(i_{1} / M, \ldots, i_{N} / M\right)$ with $i_{j} \in \mathbf{N}$. These results lead to the clustering conjecture:

If $B(\mathbf{q})$ is a continuous factor of $B(\mathbf{p})$, then $\mathbf{q}$ is a clustering of p, i.e., there is a partition $\left(I_{1}, \ldots, I_{N}\right)$ of $\{1, \ldots, M\}$ such that $q_{k}=\sum_{i \in I_{k}} p_{i}$.

This conjecture was disproved by Boyle and Tuncel [1]. They showed that $B\left(\frac{1}{3}, \frac{1}{3}, \frac{1}{3}\right)$ is a continuous (two-block) factor of $B\left(\frac{1}{6}, \frac{1}{6}, \frac{2}{9}, \frac{2}{9}, \frac{2}{9}\right)$. Smorodinsky [3] analyzed this example and arrived at a method for producing further counterexamples to the clustering conjecture.

In this note we present an algebraic independence condition on the $p_{i}$ which assures that $B(\mathbf{p})$ obeys the clustering conjecture.

Suppose now that $\mathbf{p}=(\overbrace{\pi_{1}, \ldots, \pi_{1}}^{u_{1} \text {-times }}, \overbrace{\pi_{2}, \ldots, \pi_{2}}^{u_{2} \text {-times }}, \ldots, \overbrace{\pi_{n}, \ldots, \pi_{n}}^{u_{n} \text {-times }}), \sum_{i} u_{i}$ $=M$, and let $P=\mathbf{p}^{\mathrm{Z}}, \sigma=\sigma_{M}$. Set $\lambda_{i}=\pi_{i} / \pi_{1}, i=1, \ldots, n$, and note that $\pi_{1}^{-1}=u_{1}+\sum_{i=2}^{n} \lambda_{i} u_{i}$. Fix $s \in\{1, \ldots, N\}$ (the state space of $B(\mathbf{q})$ )

Received by the editors February 23, 1987.

1980 Mathematics Subject Classification (1985 Revision). Primary 28D99. 
and let $A=A(s)=\Phi^{-1}\left({ }_{0}[s]_{0}\right)$, the set of all bisequences over $\{1, \ldots, M\}$ whose images under $\Phi$ have $S$ as its 0 -coordinate. Since $\Phi$ is continuous, there are $r_{1}, r_{2} \in \mathbf{N}$ such that $A$ is the disjoint union of cylinders $\{\omega \in$ $\left.\{1, \ldots, M\}^{\mathbf{Z}} ; \omega_{i}=x_{i},-r_{1} \leq i \leq r_{2}\right\}$ for certain $x_{i} \in\{1, \ldots, M\}$. Let $r=r_{1}+r_{2}+1$ and $P_{k}(A)=P\left(A \cap \sigma^{-1} A \cap \cdots \cap \sigma^{-(k-1)} A\right), k \in \mathbf{N} . P_{k}(A)$ can be written as

$$
P_{k}(A)=\sum_{\mathbf{j}} a_{k}(\mathbf{j}) \cdot \pi_{1}^{j_{1}} \cdots \pi_{n}^{j_{n}},
$$

where the sum extends over all $n$-tuples $\mathbf{j}=\left(j_{1}, \ldots, j_{n}\right)$ of nonnegative integers with $\sum_{\nu} j_{\nu}=k+r-1$. The coefficients $a_{k}(\mathbf{j})$ are nonnegative integers, too.

Extracting $\pi_{1}^{k+r-1}$ this can be rewritten as

$$
P_{k}(A)=\pi_{1}^{k+r-1} R_{k}\left(\lambda_{2}, \ldots, \lambda_{n}\right),
$$

where $R_{k}$ is the polynomial given by

$$
R_{k}\left(X_{2}, \ldots, X_{n}\right)=\sum_{\mathbf{j}} a_{k}(\mathbf{j}) \cdot X_{2}^{j_{2}} \cdots X_{n}^{j_{n}},
$$

the sum ranging over the same index set as above.

Since $\Phi$ is a homomorphism, $P_{k}(A)=\left(P_{1}(A)\right)^{k}$ or, equivalently,

$$
\pi_{1}^{k+r-1} R_{k}\left(\lambda_{2}, \ldots, \lambda_{n}\right)=\pi_{1}^{k r}\left(R_{1}\left(\lambda_{2}, \ldots, \lambda_{n}\right)\right)^{k},
$$

and we get the following necessary condition for $B(\mathbf{q})$ being a continuous factor of $B(\mathbf{p})$ :

$$
\frac{R_{k}\left(\lambda_{2}, \ldots, \lambda_{n}\right)}{R_{1}\left(\lambda_{2}, \ldots, \lambda_{n}\right)}=\left(\frac{R_{1}\left(\lambda_{2}, \ldots, \lambda_{n}\right)}{\left(u_{1}+\sum_{i=2}^{n} \lambda_{i} u_{i}\right)^{r-1}}\right)^{k-1}, \quad \text { for all } k .
$$

Proposition 1. Suppose that $\lambda_{2}, \ldots, \lambda_{n}$ are algebraically independent. If $B(\mathbf{q})$ is a continuous factor of $B(\mathbf{p})$, then $\mathbf{q}$ is a clustering of $\mathbf{p}$.

Proof. In this case (1) is equivalent to the corresponding polynomial identities with $\lambda_{i}$ replaced by $X_{i}$. As the polynomials in $n-1$ variables over the integers constitute a unique factorization domain and as the linear polynomial $\left(u_{1}+\sum_{i=2}^{n} X_{i} u_{i}\right)$ is irreducible over $\mathbf{Q}\left[X_{2}, \ldots, X_{n}\right]$, it follows that $\left(u_{1}+\sum_{i=2}^{n} X_{i} u_{i}\right)^{r-1}$ divides $R_{1}\left(X_{2}, \ldots, X_{n}\right)$. Hence there is a polynomial $S\left(X_{2}, \ldots, X_{n}\right)$ over $\mathbf{Z}$ such that

$$
R_{1}\left(X_{2}, \ldots, X_{n}\right)=S\left(X_{2}, \ldots, X_{n}\right) \cdot\left(u_{1}+\sum_{i=2}^{n} u_{i} X_{i}\right)^{r-1} .
$$

Since $R_{1}$ is of degree $\leq r, S\left(X_{2}, \ldots, X_{n}\right)=c_{1}+\sum_{i=2}^{n} c_{i} X_{i}$ for some $c_{i} \in \mathbf{Z}$, and, comparing the coefficients on both sides of (2), one gets

$$
n_{A}(i):=a_{1}(0, \ldots, 0, r, 0, \ldots, 0)=c_{i} u_{i}^{r-1}, \quad i=1, \ldots, n,
$$


(the $r$ occurs at the $i$ th position). $n_{A}(i)$ is just the number of blocks over the $r$ coordinates $-r_{1}, \ldots, r_{2}$ which are contained in $A$ and all of whose entries have probability $\pi_{i}$. Hence $c_{i}=c_{i}(A)=n_{A}(i) u_{i}^{-(r-1)}$ is a nonnegative integer,

$$
\begin{aligned}
P(A) & =\pi_{1}^{r} R_{1}\left(\lambda_{2}, \ldots, \lambda_{n}\right) \\
& =\pi_{1}\left(c_{1}+\sum_{i=2}^{n} c_{i} \lambda_{i}\right) \pi_{1}^{r-1}\left(u_{i}+\sum_{i=2}^{n} u_{i} \lambda_{i}\right)^{r-1} \\
& =\sum_{i=1}^{n} c_{i} \pi_{i},
\end{aligned}
$$

and, for each fixed $i=1, \ldots, n$,

$$
\sum_{s=1}^{N} c_{i}(A(s))=u_{i}^{-(r-1)} \sum_{s=1}^{N} n_{A(s)}(i)=u_{i}
$$

Remark. If $\mathbf{p}=(1 / M, \ldots, 1 / M)$, then (1) reduces to the key observation in the proof of the clustering conjecture for this case as given by del Junco et al. [2].

Note added in proof. Recently, S. Tuncel [Ergodic Theory Dynamical Systems 9 (1989), 561-570] proved a considerable extension of this result to the case where only the transcendental elements from $\left\{\lambda_{2}, \ldots, \lambda_{n}\right\}$ are assumed to be algebraically independent. This includes the case where all $\lambda_{i}$ are algebraic.

\section{REFERENCES}

1. M. Boyle and S. Tuncel, Infinite-to-one codes and Markov measures, Trans. Amer. Math. Soc. 285 (1984), 657-684.

2. A. del Junco, M. Keane, B. Kitchens, B. Marcus, and L. Swanson, Continuous homomorphisms of Bernoulli schemes, Ergodic theory and dynamical systems I, Progress in Mathematics, vol. 10, Birkhäuser, Boston, Basel, and Stuttgart, 1981, pp. 91-111.

3. M. Smorodinsky, Block codes for Bernoulli shifts, Israel J. Math. 49 (1984), 325-330.

4. S. Tuncel, Conditional pressure and coding, Israel J. Math. 39 (1981), 101-112.

Sonderforschungsbereich 123, Universität Heidelberg, D-6900 Heidelberg, West GerMANY

Current address: Mathematisches Institut, Universität Erlangen-Nürnberg, Bismarckstr. $1 \frac{1}{2}$, D-8520 Erlangen, West Germany 